\title{
Light absorption in the near field around surface plasmon polaritons
}

\author{
Antonio Luque, Antonio Martí, Manuel J. Mendes, ${ }^{\text {a) }}$ and Ignacio Tobías \\ Instituto de Energia Solar, Universidad Politecnica de Madrid, E28040 Madrid, Spain
}

(Received 5 June 2008; accepted 22 September 2008; published online 12 December 2008)

\begin{abstract}
A semiclassical method is developed to calculate the energy absorption of an electronic system located in the near field of a metal nanoparticle sustaining surface plasmons. The results are found to be similar to those of photon absorption from ordinary transversal radiation. However, they are affected by a geometrical factor that can increase the absorption by several orders of magnitude. As example, we investigate ellipsoidal-shaped metal nanoparticles which, under favorable conditions, may provide near field aborption enhancements almost as large as $10^{4}$, and in many cases above 10 . (C) 2008 American Institute of Physics. [DOI: 10.1063/1.3014035]
\end{abstract}

\section{INTRODUCTION}

The radiation absorption and emission mechanisms in the interaction between light and a material system, whose foundations were attributed to Dirac by Fermi ${ }^{1}$ in 1932, have been extensively presented in textbooks. ${ }^{2-4}$ This interaction is produced between the electrons of the material system (atom or quantum dot, for instance) and a transversal radiation, that is, derived from a vector potential of zero divergence (and this is an important condition ${ }^{5}$ ). In addition, boundary conditions can modify the radiation modes and, consequently, the response of the electronic system.

Significant attention has been paid to the modifications of the spontaneous emission ${ }^{6,7}$ as a result of confined light since the pioneering work of Purcell $^{8}$ in 1946. The possibilities of suppressing or enhancing the emitted radiation in novel structures such as photonic crystals, ${ }^{9,10}$ or more recently plasmonic devices, ${ }^{11-15}$ has attracted the interest of many workers.

On the other hand, when radiation falls on a metal nanoparticle (MNP) it excites oscillations of the free electron plasma, whose quanta are called surface plasmon polaritons (SPPs) since these modes are confined within the nanoparticle surface. ${ }^{16,17}$ A spherical scattered wave far from the particle will appear that can be large when the frequency of the incident wave is close to the plasma resonance frequency. ${ }^{18,19}$ However, in the present work we are mostly interested in the scattered near field which fulfills the boundary conditions imposed by Maxwell equations for the electromagnetic field $^{18,20}$ (Sec. 13.5 in Ref. 18) at the MNP surface. Contrary to the incoming electromagnetic radiation, this field is essentially (but not uniquely) a longitudinal electric field of zero curl (derived from a scalar potential) which can be very strong near the resonance.

The interaction of this near field with the electrons of the material, in which the MNP is embedded, cannot be solved using the ordinary formulas of emission and absorption of photons in the radiation modes given in the classic text books referenced above. This is because the traditional derivations only deal with the quantification of transversal fields,

${ }^{\text {a)} E l e c t r o n i c ~ m a i l: ~ m a n u e l . m e n d e s @ i e s-d e f . u p m . e s . ~}$ and the same treatment cannot be applied, in principle, for a longitudinal perturbation such as the case of the MNP near field.

As matter of fact, while a lot of effort has recently been made in the analysis of the spontaneous emission in special structures (and of course of stimulated emission for lasers) much less effort has been made, to our knowledge, in the study of the energy absorption by atoms soaked in the longitudinal field around the MNP sustaining plasmonic oscillations. The formulas to apply to this case are obtained in this paper.

To address this problem, a semiclassical approach is employed that considers the near field created by the ordinary radiation near (but outside) the MNP as an external classic field, not affected by the interaction with the material system consisting of the atoms or quantum dot in the surrounding medium. To verify this procedure, the same method is applied to the ordinary radiation (that in Fermi's work) to ascertain how it differs from the quantum case with electromagnetic field quantification and interaction of the field with matter and vice versa. Once the semiclassical method is evaluated, its application to the longitudinal field is solved; and finally the action of the matter on the longitudinal field, associated with the emission of energy into the near field, is obtained employing detailed balance arguments.

The procedure is applied and discussed for the longitudinal field sustained by the SPP modes in ellipsoidal MNPs. This allows us to calculate the absorption improvement which is actually strongly dependent on the radiation wavelength and the shape of the particle.

Although the results of this paper are expected to be applicable in different areas, much interest has recently been raised $^{21-26}$ to its implementation in solar cells. In this respect, the authors plan to consider the application of SPPs to intermediate band solar cells ${ }^{27,28}$ (IBSCs) and, in particular, to those manufactured with quantum dots. ${ }^{29,30}$ This is motivated by the high efficiency potential of IBSCs. In the radiative limit, these cells show a limiting efficiency of $63.2 \%$ to be compared to $40.7 \%$ of single gap solar cells calculated in the same conditions. Experimental quantum dot IBSCs have already allowed the verification of the principles of operation of the IBSC concept. ${ }^{31,32}$ However, the impact of the inter- 
mediate band effects on the cell performance is still marginal, mainly due to the weak absorption of light provided by the quantum dots. ${ }^{33}$ The absorption coefficient of belowband-gap photons in these cells is quite small, more than one order of magnitude lower than the one of above-band-gap photons. ${ }^{30}$ The insertion of MNPs close to the quantum dots is a promising way to increase this absorption. This process has already been shown to amplify quantum dot photoluminescence, ${ }^{34}$ similar to the well-known effect of emission enhancement from fluorescence molecules when placed in the vicinity of metal surfaces. ${ }^{35}$

This paper is organized as follows. We first present the background concerning how the formulas for the absorption and emission of phonons are obtained traditionally, ${ }^{1,3,36}$ for a weakly absorbing medium characterized by a purely real dielectric constant. The SI system of units is used. In Sec. III, the semiclassical model is derived for classic radiation and compared to the purely quantum theory. Then the model is determined for the near field and discussed for the case of an ellipsoidal MNP illuminated by ordinary radiation which excites the SPP resonance in the particle. A discussion of the results is included.

Other configurations of MNP could also be of interest besides the ones presented here (e.g., couples of MNP such as those discussed in Ref. 12). As such, without discounting the interest in analyzing the selected MNP structure, the main purpose of this paper is to provide a tool for the analysis of the absorption in the presence of any (including others possibly more advanced) MNP structure.

\section{BACKGROUND}

\section{A. Quantification of the free radiation}

As previously referred, the development of free radiation into photons is well known. In the Coulomb or radiation gauge, characterized by setting $\nabla \cdot \boldsymbol{A}=0$, the electromagnetic field can be derived entirely from a vector potential so that $\boldsymbol{B}=\nabla \times \boldsymbol{A}$ and $\boldsymbol{E}=-\partial \boldsymbol{A} / \partial t$. To simplify the reasoning we consider that the radiation is confined to a volume $V$ very large but finite, and we restrict our treatment to nonmagnetic materials $(\mu=1)$.

The vector potential can be developed in a set of orthogonal functions of the space coordinates (plane waves in our work) so that

$$
\begin{aligned}
& \boldsymbol{A}(\boldsymbol{r}, t)=\sum_{i=1,2, \boldsymbol{k}} \hat{A}_{i}(\boldsymbol{k}, t) \cdot \boldsymbol{\tau}_{i}(\boldsymbol{k}) \exp (i \boldsymbol{k} \cdot \boldsymbol{r}), \\
& \hat{A}_{i}(\boldsymbol{k}, 0)=\int \boldsymbol{A}(\boldsymbol{r}, 0) \cdot \boldsymbol{\tau}_{i}(\boldsymbol{k}) \exp (-i \boldsymbol{k} \cdot \boldsymbol{r}) d \boldsymbol{r} / V,
\end{aligned}
$$

where $\boldsymbol{\tau}_{i}(\boldsymbol{k})$ are the polarization unity vectors that have to be normal to $\boldsymbol{k}\left(\nabla\left[\boldsymbol{\tau}_{i} \exp (i \boldsymbol{k} \cdot \boldsymbol{r})\right]=i \boldsymbol{k} \cdot \boldsymbol{\tau}_{i} \exp (i \boldsymbol{k} \cdot \boldsymbol{r})=0\right)$ as well as orthonormal $\boldsymbol{\tau}_{i}(\boldsymbol{k})^{*} \boldsymbol{\tau}_{j}(\boldsymbol{k})=\delta_{i j}$. Note also that the time dependence is in the Fourier series coefficients $\hat{A}_{i}(\boldsymbol{k}, t)$, and for $\boldsymbol{A}(\boldsymbol{r}, t)$ to be real, $\hat{A}_{i}(-\boldsymbol{k}, t)=\hat{A}_{i}^{*}(\boldsymbol{k}, t)$.

The vector potential follows the electromagnetic wave equation so that

$$
\begin{aligned}
& \sum_{i=1,2 ; \mathbf{k}} k^{2} \hat{A}_{i}(\boldsymbol{k}, t) \cdot \boldsymbol{\tau}_{i}(\boldsymbol{k}) \exp (i \boldsymbol{k} \cdot \boldsymbol{\tau}) \\
& \quad+\left(n^{2} / c^{2}\right) \sum_{i=1,2 ; \mathbf{k}}\left[\partial^{2} \hat{A}_{i}(\boldsymbol{k}, t) \cdot \boldsymbol{\tau}_{i}(\boldsymbol{k}) / \partial t^{2}\right] \exp (i \boldsymbol{k} \cdot \mathbf{r})=0
\end{aligned}
$$

Hence,

$$
\begin{aligned}
\frac{\partial^{2} \hat{A}_{i}(\boldsymbol{k}, t)}{\partial t^{2}}+\frac{k^{2} c^{2}}{n^{2}} \hat{A}_{i}(\boldsymbol{k}, t) & =0 \quad \text { with } k c / n=\omega, \\
& \Rightarrow \hat{A}(\boldsymbol{k}, t)=\hat{A}(\boldsymbol{k}, 0) \exp (-i \omega t) .
\end{aligned}
$$

The energy contained in the electromagnetic field is $U$ $=(1 / 2) \int\left(\varepsilon \varepsilon_{0} E^{2}+\mu \mu_{0} H^{2}\right) d r$. When the fields are harmonic and represented by complex vectors, the time average of the energy is given by $\langle U\rangle=\operatorname{Re}\left[(1 / 2) \int\left(\varepsilon \varepsilon_{0} \mathbf{E}^{*} \cdot \mathbf{E}\right.\right.$ $\left.\left.+\mu \mu_{0} \mathbf{H}^{*} \cdot \mathbf{H}\right) d r\right] / 2$. Using the electromagnetic field expansion in Eq. (1), the average energy is (for $\mu=1$ and real $\varepsilon$ $=n^{2}$, implying weak absorption)

$$
\begin{aligned}
\langle U\rangle & =\sum_{i, \mathbf{k}} \frac{V(\omega n)^{2} \varepsilon_{0}}{2} \hat{A}_{i}^{*}(\boldsymbol{k}, 0) \hat{A}_{i}(\boldsymbol{k}, 0) \\
& =\sum_{i, \mathbf{k}} \frac{V(\omega n)^{2} \varepsilon_{0}}{2} \frac{\hat{A}_{i}(\boldsymbol{k}, 0) \hat{A}_{i}^{*}(\boldsymbol{k}, 0)+\hat{A}_{i}^{*}(\boldsymbol{k}, 0) \hat{A}_{i}(\boldsymbol{k}, 0)}{2} .
\end{aligned}
$$

In treating the complete quantum mechanical problem, it is useful to express the average energy $\langle U\rangle$ in terms of the radiation Hamiltonian given by an appropriate set of creation and annihilation operators,

$$
\begin{aligned}
\langle U\rangle & \Rightarrow \mathcal{H}_{\mathrm{rad}}=\sum_{i, \mathbf{k}} \hbar \omega \frac{a_{i}(\boldsymbol{k}) a_{i}^{+}(\boldsymbol{k})+a_{i}^{+}(\boldsymbol{k}) a_{i}(\boldsymbol{k})}{2} \\
& =\sum_{i, k} \mathcal{H}_{\mathrm{rad}, i}(\boldsymbol{k}),
\end{aligned}
$$

where the (dimensionless) creation and annihilation operators $a_{i}^{+}(\boldsymbol{k})$ and $a_{i}(\boldsymbol{k})$ are noncommulative, that is, $\left[a_{i}(\boldsymbol{k}) a_{i}^{+}(\boldsymbol{k})\right]=1$. Setting this condition is the essence of the field quantification. The arrow means a rule of correspondence.

The immediate consequence of the preceding is that the vector potential Fourier components are directly associated to the annihilation and creation operators (through a constant that respects the magnitude dimensions),

$$
\begin{aligned}
& \hat{A}_{i}(\boldsymbol{k}, 0)=\left(\frac{1}{n}\right)\left(\frac{2 \hbar}{\omega V \varepsilon_{0}}\right)^{1 / 2} a_{i}(\boldsymbol{k}), \\
& \hat{A}_{i}^{*}(\boldsymbol{k}, 0)=\left(\frac{1}{n}\right)\left(\frac{2 \hbar}{\omega V \varepsilon_{0}}\right)^{1 / 2} a_{i}^{+}(\boldsymbol{k}),
\end{aligned}
$$

and that the use of the commutator relates the quantum Hamiltonian to the number of particles operators: $N_{i}(\boldsymbol{k})$ $=a_{i}^{+}(\boldsymbol{k}) a_{i}(\boldsymbol{k})$,

$$
\mathcal{H}_{\mathrm{rad}, i}(\boldsymbol{k})=\hbar \omega\left[N_{i}(\boldsymbol{k})+\frac{1}{2}\right] \text {. }
$$


The eigenvectors of the number of particles operators have important properties (Vol. I, p. 370 in Ref. 3). Let $\left|\nu \boldsymbol{k} \tau_{i}\right\rangle$ be an eigenvector of $N$ of eigenvalue $\nu$ and norm unity. In this case, $a_{i}^{+}(\boldsymbol{k})\left|\nu \boldsymbol{k} \boldsymbol{\tau}_{i}\right\rangle=(\nu+1)^{1 / 2}\left|(\nu+1) \boldsymbol{k} \boldsymbol{\tau}_{i}\right\rangle$ and $a_{i}(\boldsymbol{k})\left|\nu \boldsymbol{k} \boldsymbol{\tau}_{i}\right\rangle$ $=(\nu)^{1 / 2}\left|(\nu-1) \boldsymbol{k} \boldsymbol{\tau}_{i}\right\rangle$. Furthermore, $a_{i}(\boldsymbol{k})\left|0 \boldsymbol{k} \boldsymbol{\tau}_{i}\right\rangle=0$. The values of $\nu$ are integer and they represent the number of particles, in this case photons, in the mode $\boldsymbol{k}, \boldsymbol{\tau}_{i}$. If necessary we can write $\nu_{i}(\boldsymbol{k})$ or $\nu_{k, i}$.

\section{B. Matter-field interaction Hamiltonian}

If the coupling of the magnetic field with the spin is neglected, the nonrelativistic Hamiltonian of one charged particle in the presence of electromagnetic fields reflects the invariance of the norm of the quadrivector energy impulsion in presence of such a field. Once the rest mass energy of the particle is subtracted (Vol II, p. 807 in Ref. 3), the Hamiltonian is

$$
\begin{aligned}
\mathcal{H}_{\text {particle }}= & \frac{(\boldsymbol{p}-q \boldsymbol{A})^{2}}{2 m}+q \Phi \cong \frac{p^{2}}{2 m}-\frac{q}{2 m}(\boldsymbol{p} \cdot \boldsymbol{A}+\boldsymbol{A} \cdot \boldsymbol{p}) \\
& +q \Phi .
\end{aligned}
$$

For the case of a set of electrons, this becomes

$$
\begin{aligned}
\mathcal{H}_{\text {mat }} & =\sum_{j} \frac{p_{j}^{2}}{2 m}-e \varphi, \\
\mathcal{H}_{\text {int }} & =\sum_{j}\left(\frac{e}{2 m}\left[\boldsymbol{p}_{j} \cdot \boldsymbol{A}\left(\boldsymbol{r}_{j}\right)+\boldsymbol{A}\left(\boldsymbol{r}_{j}\right) \cdot \boldsymbol{p}_{j}\right]-e \boldsymbol{\phi}\left(\boldsymbol{r}_{j}\right)\right) \\
& =\sum_{j} \mathcal{H}_{\mathrm{int}, i} .
\end{aligned}
$$

In the first formula of the matter Hamiltonian the scalar potential $\varphi$ is considered as made up of all the nuclei and electrons (and can possibly be decomposed into terms $\varphi_{j}$ following the general methods of solid state physics). In the second expression of the interaction Hamiltonian, $\phi$ is the scalar potential corresponding to the longitudinal field around the MNP where the SPP is excited. $\boldsymbol{A}$ is the vector potential from the incoming radiation field.

\section{Photon absorption and emission}

The interaction of matter with free radiation is described by the interaction Hamiltonian in Eq. (8) with $\phi\left(\boldsymbol{r}_{j}\right)=0$. Taking into account that (see Vol. I, p. 175 in Ref. 3) $\left[p_{x}, F\left(x, p_{x}\right)\right]=-i \hbar \partial F / \partial x$, and that $\boldsymbol{A}$ is transversal, $\boldsymbol{p}_{j} \cdot \boldsymbol{A}$ $-\boldsymbol{A} \cdot \boldsymbol{p}_{j}=-i \hbar \nabla \boldsymbol{A}=0$, so that $\boldsymbol{A}$ commutes with $\boldsymbol{p}_{j}$ and $\mathcal{H}_{\text {int, }, j}$ $=e \boldsymbol{p}_{j} \cdot \boldsymbol{A}\left(\boldsymbol{r}_{j}, t\right) / \mathrm{m}$.

Let us assume an initial state $\left|\lambda, \nu \boldsymbol{k} \boldsymbol{\tau}_{i}\right\rangle$ and a final state $\left|\mu,(\nu+1) k \tau_{i}\right\rangle$. The two states differ only in the electronic state $(\lambda, \mu)$ and in that there is one more photon in a photonic mode (photon emission). The Fermi golden rule (Vol. II, p. 631 in Ref. 3) establishes that the probability of transition per unit of time between these states, caused by a timeindependent Hamiltonian, is

$$
\begin{aligned}
w_{\lambda, \nu k \tau_{i} \rightarrow \mu,(\nu+1) k \tau_{i}}= & \frac{2 \pi}{\hbar} \mid\left\langle\lambda, \nu \boldsymbol{k} \tau_{i}\left|\mathcal{H}_{\text {int }}\right| \mu,(\nu\right. \\
& \left.+1) \boldsymbol{k} \tau_{i}\right\rangle\left.\right|^{2} \delta\left(\mathfrak{E}_{\mu,(\nu+1) \boldsymbol{k} \tau_{i}}-\mathfrak{E}_{\lambda, \nu k \tau_{i}}\right),
\end{aligned}
$$

where $\epsilon$ is the energy (of the matter-radiation system or of the electron system, depending on subindices). A similar expression is used for the photon absorption $\left|\mu, \nu k \tau_{i}\right\rangle \rightarrow \mid \lambda,(\nu$ $\left.-1) \boldsymbol{k} \boldsymbol{\tau}_{i}\right\rangle$.

The vector potential of the interaction Hamiltonian must be considered as the sum of its Fourier components including operators proportional to the annihilation and creation operators that act on the space of photons, not on the electrons. Taking into account that (Vol. I, p. 175 in Ref. 3) $\left[x, F\left(x, p_{x}\right)\right]=i \hbar \partial F / \partial p_{x}$, we can write that $\left\lfloor\boldsymbol{r}_{j}, \mathcal{H}_{\text {mat }}\right\rfloor=i \hbar \boldsymbol{p}_{j} / m$ and, for $\exp (i k r) \cong 1$ (radiation wavelengths much larger than the electronic wavefunctions), the matrix element in Eq. (9) can be written as

$$
\begin{aligned}
\left\langle\lambda, \nu \boldsymbol{k} \boldsymbol{\tau}_{i}\right| & \frac{e}{m_{i, \boldsymbol{k}, j}} \boldsymbol{p}_{j} \cdot \boldsymbol{\tau}_{i} \\
& \times\left[\frac{\hat{A}_{i}(\boldsymbol{k}, 0) \exp (i \boldsymbol{k r})+\hat{A}_{i}^{*}(\boldsymbol{k}, 0) \exp (-i \boldsymbol{k r})}{2}\right] \mid \mu,(\nu \\
+ & \left.1) \boldsymbol{k} \boldsymbol{\tau}_{i}\right\rangle=\left(\frac{2}{2 i \hbar n}\right)\left(\frac{2 \hbar}{\omega V \varepsilon_{0}}\right)^{1 / 2}\left\langle\nu \boldsymbol{k} \boldsymbol{\tau}_{i}\right| \sum_{i, \boldsymbol{k}} a_{i}(\boldsymbol{k}) \\
+ & a_{i}^{+}(\boldsymbol{k})\left|(\nu+1) \boldsymbol{k} \boldsymbol{\tau}_{i}\right\rangle\left\langle\lambda\left|\sum_{i, j}\left[\boldsymbol{r}_{j}, \mathcal{H}_{\mathrm{mat}}\right] \cdot \boldsymbol{\tau}_{i}\right| \mu\right\rangle \\
= & \left(\frac{e}{2 i n}\right)\left(\frac{2 \hbar}{\omega V \varepsilon_{0}}\right)^{1 / 2}\left(\frac{\mathfrak{E}_{\mu}-\mathfrak{E}_{\lambda}}{\hbar}\right)\left(\nu_{\boldsymbol{k}}\right. \\
& +1)^{1 / 2}\left\langle\lambda\left|\sum_{j} \boldsymbol{r}_{j} \cdot \boldsymbol{\tau}_{i}\right| \mu\right\rangle,
\end{aligned}
$$

where we have taken care (since the sum is real) to write only the real part of the Fourier components to ensure that the radiation operators are Hermitian for any mode. Thus, taking into account that for the transitions permitted by the Dirac delta $\left(\epsilon_{\mu}-\epsilon_{\lambda}\right) / \hbar=\omega$, the photon emission rate is

$$
\begin{aligned}
w_{\lambda, i k \tau_{i} \rightarrow \mu,(\nu+1) k \tau_{i}}= & \frac{\pi e^{2} \omega}{n^{2} V \varepsilon_{0}}\left(\nu_{\boldsymbol{k}}+1\right) \\
& \times\left|\left\langle\lambda\left|\sum_{j} \boldsymbol{r}_{j} \cdot \boldsymbol{\tau}_{i}\right| \mu,\right\rangle\right|^{2} \delta\left(\mathfrak{E}_{\mu,(\nu-1) k \tau_{i}}\right. \\
& \left.-\mathfrak{E}_{\lambda, \nu k \tau_{i}}\right),
\end{aligned}
$$

and for the absorption,

$$
\begin{aligned}
w_{\mu, \nu k \tau_{i} \rightarrow \lambda,(\nu-1) k \tau_{i}}= & \frac{\pi e^{2} \omega}{n^{2} V \varepsilon_{0}} \nu_{k}\left|\left\langle\lambda\left|\sum_{j} \boldsymbol{r}_{j} \cdot \tau_{i}\right| \mu,\right\rangle\right|^{2} \\
& \times \delta\left(\mathfrak{E}_{\mu, \nu k \tau_{i}}-\mathfrak{E}_{\lambda,(\nu-1) k \tau_{i}}\right) .
\end{aligned}
$$

In these formulas, the arbitrary volume $V$, introduced for simplicity, appears. Since the emission does not occur in a single photon mode but rather in a multiplicity of modes of density per unit of energy (p. 115 in Ref. 36) $n^{3} V \epsilon^{2} / \pi^{2} \hbar^{3} c^{3}$, after integration of the probability of emission on all the 
possible modes, the volume disappears (as well as the Dirac delta). The same can be said for the absorption, since the matter is actually illuminated by a nonmonochromatic bundle with a certain angular extension. Furthermore, additional integration is required by the (joint) density of electronic states per unit of volume that finally makes all these transitions (in the limit of weak absorption) to be proportional to the volume of the material system which is actually illuminated. These details are not developed here because they are not necessary for the purposes of this paper.

\section{SEMICLASSICAL RADIATION AND EMISSION MODELS}

\section{A. Radiation absorption and emission}

In the semiclassical approach developed in this section we consider the radiation to be described by a classical potential vector, such as the one in Eq. (1), and with a time evolution as the one shown in Eq. (2), harmonic for each term that is calculated following a classical (non quantum) pattern. In this section the method is evaluated by comparing it with the well-established results given in Eqs. (11) and (12) for a transversal field. Therefore, for now we only account for the interaction of matter with free radiation, that is, the interaction-scalar potential $\phi$ present in Eq. (8) is not considered for the moment. Furthermore, the vector potential is transversal so that $\boldsymbol{A}$ commutes with $\boldsymbol{p}_{j}$, as does each $\boldsymbol{A}$ term. The interaction Hamiltonian is, as above, $\mathcal{H}_{\text {int, } j}$ $=e \boldsymbol{p}_{j} \cdot \boldsymbol{A}\left(\boldsymbol{r}_{j}, t\right) / m, \boldsymbol{A}$ now being a function of the space and time consisting of a series of harmonic terms.

The Fermi golden rule for the case of harmonic timedependent operators of the type $\left[V \exp (-i \omega t)+V^{+} \exp (i \omega t)\right] / 2$ takes a slightly different aspect than that in Eq. (9). It is (p. 559 in Ref. 37)

$$
\begin{aligned}
w_{\lambda \rightarrow \mu}= & \frac{\pi}{2 \hbar}\left\{|\langle\lambda|V| \mu\rangle|^{2} \delta\left(\mathfrak{E}_{\lambda}-\mathfrak{E}_{\mu}-\hbar \omega\right)+|\langle\lambda|V| \mu\rangle|^{2} \delta\left(\mathfrak{E}_{\lambda}\right.\right. \\
& \left.\left.-\mathfrak{E}_{\mu}+\hbar \omega\right)\right\}
\end{aligned}
$$

where only one term may be non-null. The term on the left can be interpreted as a photon emission and the term on the right as a photon absorption.

Let us assume an initial state $\left|\lambda, \boldsymbol{k} \boldsymbol{\tau}_{i}\right\rangle$ and a final state $\left|\mu, \boldsymbol{k} \boldsymbol{\tau}_{i}\right\rangle$. No mention of the number of photons is contained in this semiclassical nomenclature, but we note that the radiation corresponds to a single mode $\boldsymbol{k} \boldsymbol{\tau}_{i}$. Using all of the transformations already applied for the purely quantum case in the previous section, we obtain for the emissions and absorptions (super indices $e$ and $a$ )

$$
\begin{aligned}
w_{\lambda, \boldsymbol{k} \tau_{i} \rightarrow \mu, \boldsymbol{k} \tau_{i}}^{(e)}= & w_{\mu, \boldsymbol{k} \tau_{i} \rightarrow \lambda, \boldsymbol{k} \tau_{i}}^{(a)} \\
= & \frac{\pi e^{2} \omega^{2}}{2 \hbar}\left|\hat{A}_{i}(\boldsymbol{k}, 0)\right|^{2}\left|\left\langle\lambda\left|\sum_{j} \boldsymbol{r}_{j} \cdot \boldsymbol{\tau}_{i}\right| \mu\right\rangle\right|^{2} \delta\left(\mathfrak{E}_{\lambda}\right. \\
& \left.-\mathfrak{E}_{\mu}-\hbar \omega\right) .
\end{aligned}
$$

Note that contrary to the quantum model, the semiclassical model absorption and emission probability are the same and proportional to the vector potential.
Let us now relate the magnitude of the vector potential with the number of photons in the mode. One mode filled with $\nu_{k}$ photons has a density of $\nu_{k} / V$ photons $/ \mathrm{m}^{3}$ and carries a power density in the direction of the vector $\boldsymbol{k}$ of magnitude $\nu_{k} \hbar \omega c / V n$. This quantity can be expressed as a function of the electric field associated to these photons by writing the time average Poynting vector (p. 33 in Ref. 18) $\langle\boldsymbol{S}\rangle=(1 / 2) \operatorname{Re}\left[\hat{\boldsymbol{E}} \times \hat{\boldsymbol{H}}^{*}\right]$, that for a plane wave is in the propagation vector direction (p. 24 in Ref. 18) and takes the value $\langle S\rangle=(1 / 2) \sqrt{\varepsilon \varepsilon_{0} / \mu \mu_{0}}\left|\hat{E}_{i}(\boldsymbol{k}, 0)\right|^{2}=\left(n / 2 \mu_{0} c\right)\left|\hat{E}_{i}(\boldsymbol{k}, 0)\right|^{2}$ (the last equality for $\mu=1$ and $\left.\varepsilon=n^{2}\right)$. Thus,

$$
\left|\hat{E}_{i}(\boldsymbol{k}, 0)\right|^{2}=2 \nu_{\boldsymbol{k}} \hbar \omega / V n^{2} \varepsilon_{0} .
$$

To calculate the value of the vector potential associated to this photon density we take into account that for transversal waves, $\boldsymbol{E}=-\partial \boldsymbol{A} / \partial t=-i \omega \boldsymbol{A}$ and obtain $\langle S\rangle$ $=\left(n \omega^{2} / 2 \mu_{0} c\right)\left|\hat{A}_{i}(\boldsymbol{k}, 0)\right|^{2}$. Consequently,

$$
\left|\hat{A}_{i}(\boldsymbol{k}, 0)\right|^{2}=2 \nu_{\boldsymbol{k}} \hbar / V \omega n^{2} \varepsilon_{0} .
$$

We can now write the emission probability in Eq. (14) as

$$
\begin{aligned}
w_{\lambda, k}^{(e)} \tau_{i} \rightarrow \mu, k \tau_{i} & =\frac{\pi e^{2} \omega}{V n^{2} \varepsilon_{0}} \nu_{k}\left|\left\langle\lambda\left|\boldsymbol{\tau}_{i} \sum_{j} \boldsymbol{r}_{j}\right| \mu\right\rangle\right|^{2} \delta\left(\mathfrak{E}_{\lambda}-\mathfrak{E}_{\mu}\right. \\
& -h \omega),
\end{aligned}
$$

which is the same as Eq. (11), considering only the term of stimulated emission but without the one of spontaneous emission.

For absorptions, this model reproduces Eq. (12),

$$
\begin{aligned}
w_{\mu, k \tau_{i} \rightarrow \lambda, k \tau_{i}}^{(a)}= & \frac{\pi e^{2} \omega}{V n^{2} \varepsilon_{0}} \nu_{k}\left|\left\langle\lambda\left|\boldsymbol{\tau}_{i} \sum_{j} \boldsymbol{r}_{j}\right| \mu\right\rangle\right|^{2} \delta\left(\mathfrak{E}_{\mu}-\mathfrak{E}_{\lambda}\right. \\
& +h \omega) .
\end{aligned}
$$

Therefore, the semiclassical method leads to the same results as the full quantum method except that it neglects the spontaneous emission rate.

Materials are soaked in a gas of photons. The probability of having a mode occupied is $[\exp (\epsilon / k T)-1]^{-1}$. For photons of $1 \mathrm{eV}$ from the sun [one of the optimal band gaps in GaAs IBSCs (Ref. 27)] at about $6000 \mathrm{~K}$, this probability is $2.14 \%$; and for photons of $0.4 \mathrm{eV}$ (another optimal band gap), it is is $27 \%$. In both cases the modes are rather empty and the probability of finding two photons in a mode is quite small. Thus, $\nu_{k}$ is not much higher than one, and therefore the term $\left(\nu_{k}\right.$ +1 ) in Eq. (11) cannot be approximated to $v_{k}$ as the result of Eq. (17). Consequently, the semiclassical method is only applicable to absorption, and it would be erroneous to apply it to emission.

\section{B. Energy absorption from the SPP longitudinal near field}

\section{Excitation of the SPP}

To fix ideas, we consider a SPP excitation sustained by an ellipsoidal-shaped MNP defined by orthogonal semiaxes $a, b$, and $c$ directed, respectively, in the $x, y$, and $z$ axes of a Cartesian coordinates set. To calculate the field scattered by the ellipsoid we make use of the electrostatic approximation 
(Chap. 5 in Ref. 19) since the MNP size is taken to be much smaller than the incoming radiation wavelength in the semiconductor medium where it is embedded. The MNP material is characterized by a frequency-dependent complex dielectric function $\varepsilon_{\mathrm{MNP}}(\omega)$.

The electric field of the incoming radiation, which excites the SPP modes, is derived from a vector potential, but within the MNP it leads to a spatially constant electric field of modulus $\hat{E}_{i}(\boldsymbol{k}, 0)$, given by Eq. (15) with a harmonic time variation. Under the electrostatic approximation, this constant field can also be derived from a linearly variable scalar potential that is given by $\phi^{(i)}=-\hat{E}_{i}(\boldsymbol{k}, 0) z$ when the wave vector $\boldsymbol{k}$ of the incoming radiation is taken in the $y$ direction, and the polarization $\boldsymbol{\tau}_{i}$ is in the $z$ direction. Generally,

$$
\phi^{(i)}=-\hat{E}_{i}(\boldsymbol{k}, 0) \boldsymbol{r} \cdot \boldsymbol{\tau}_{i} .
$$

Under these conditions the scattered field is given by a harmonic time-variable scalar potential given by (p. 144 in Ref. 19)

$$
\phi^{(s)}=\phi^{(i)} \Lambda_{c} \Psi_{c}(\xi),
$$

where $\Lambda_{c}$ is the polarizability of the MNP and $\Psi_{c}(\xi)$ is a geometrical factor, function of the elliptic coordinate $\xi$ defined below,

$$
\begin{aligned}
& \Psi_{c}(\xi)=\frac{a b c}{2} \int_{\xi}^{\infty} \frac{d q}{\left(c^{2}+q\right) f(q)}, \\
& f(q)=\left[\left(a^{2}+q\right)\left(b^{2}+q\right)\left(c^{2}+q\right)\right]^{1 / 2}, \\
& \Lambda_{c}=\frac{\left(\varepsilon-\varepsilon_{\mathrm{MNP}}\right)}{\varepsilon+L_{c}\left(\varepsilon_{\mathrm{MNP}}-\varepsilon\right)}, \\
& L_{c}=\frac{a b c}{2} \int_{0}^{\infty} \frac{d q}{\left(c^{2}+q\right) f(q)}=\Psi_{c}(0) .
\end{aligned}
$$

The problem is expressed in elliptic coordinates $(\xi, \eta, \zeta)$. These coordinates are defined as follows: For the case that $a>b>c$,

$$
\begin{aligned}
& \frac{x^{2}}{a^{2}+\xi}+\frac{y^{2}}{b^{2}+\xi}+\frac{z^{2}}{c^{2}+\xi}=1, \quad \forall-c^{2}<\xi<\infty, \\
& \frac{x^{2}}{a^{2}+\eta}+\frac{y^{2}}{b^{2}+\eta}+\frac{z^{2}}{c^{2}+\eta}=1, \quad \forall-b^{2}<\eta<-c^{2}, \\
& \frac{x^{2}}{a^{2}+\zeta}+\frac{y^{3}}{b^{2}+\zeta}+\frac{z^{2}}{c^{2}+\zeta}=1, \quad \forall-a^{2}<\xi<-b^{2},
\end{aligned}
$$

conversely,

$$
\begin{aligned}
& x^{2}=\frac{\left(a^{2}+\xi\right)\left(a^{2}+\eta\right)\left(a^{2}+\zeta\right)}{\left(b^{2}-a^{2}\right)\left(c^{2}-a^{2}\right)}, \\
& y^{2}=\frac{\left(b^{2}+\xi\right)\left(b^{2}+\eta\right)\left(b^{2}+\zeta\right)}{\left(a^{2}-b^{2}\right)\left(c^{2}-b^{2}\right)},
\end{aligned}
$$

$$
z^{2}=\frac{\left(c^{2}+\xi\right)\left(c^{2}+\eta\right)\left(c^{2}+\zeta\right)}{\left(a^{2}-c^{2}\right)\left(b^{2}-c^{2}\right)} .
$$

Obviously, in elliptic coordinates,

$$
\phi^{(i)}=-\hat{E}_{i}(\boldsymbol{k}, 0) z=-\hat{E}_{i}(\boldsymbol{k}, 0)\left(\frac{\left(c^{2}+\xi\right)\left(c^{2}+\eta\right)\left(c^{2}+\zeta\right)}{\left(a^{2}-c^{2}\right)\left(b^{2}-c^{2}\right)}\right)^{1 / 2}
$$

\section{Absorption of energy from the SPP scattered field}

In this case the perturbation $H_{\mathrm{int}, j}$ appearing in Eq. (8) is $H_{\text {int }, j}=-e \phi^{(s)}\left(\boldsymbol{r}_{j}, t\right)$; that is, the electric near field potential sustained by the SPP. It is a time-dependent perturbation given by $\phi^{(s)}(\boldsymbol{r}, t)=\phi^{(s)}(\boldsymbol{r}, 0)[\exp (-i \omega t)+\exp (i \omega t)] / 2$. For a material system with several electrons $\mathcal{H}_{\text {int }}=-e \sum_{j} \phi^{(s)}\left(\boldsymbol{r}_{j}, t\right)$. Using our semiclassical treatment described in the previous section, we apply to this Hamiltonian, given by a longitudinal time-dependent perturbation, the Fermi golden rule to determine the radiation absorption. The resulting transition rate is

$$
\begin{aligned}
w_{\mu, k \tau_{i} \rightarrow \lambda, k \tau_{i}}^{(a)}= & \frac{\pi e^{2}}{2 \hbar} \mid\left\langle\lambda|\underbrace{\sum_{j} \phi^{(i)}\left(r_{j}, 0\right) \Lambda_{c} \Psi_{c}\left(\xi_{j}\right)}_{\phi^{(s)}}| \mu\right\rangle^{2} \\
& \times \delta\left(\mathfrak{E}_{\mu}-\mathfrak{E}_{\lambda}+\hbar \omega\right) .
\end{aligned}
$$

Using the expression of $\hat{E}_{i}(k, 0)$ given by Eq. (15), and also Eq. (20) we obtain

$$
\begin{gathered}
w_{\mu, k \tau_{i} \rightarrow \lambda, k \tau_{i}}^{(a)}=\frac{\pi e^{2} \omega \nu_{k}}{V n^{2} \varepsilon_{0}}\left|\left\langle\lambda\left|\boldsymbol{\tau}_{i}^{\sum_{j} r_{j} \Lambda_{c} \Psi_{c}\left(\xi_{i}\right)}\right| \mu\right\rangle\right|^{2} \\
\times \delta\left(\mathfrak{E}_{\mu}-\mathfrak{E}_{\lambda}+\hbar \omega\right) .
\end{gathered}
$$

This result differs from the absorption rate of Eq. (18) only in the extra factor $\Lambda_{c} \Psi_{c}\left(\xi_{i}\right)$ in the integrand of the matrix element, a factor that comes from the field scattered by the MNP [Eq. (20)]. This scattering factor can increase the probability of transition by several orders of magnitude in the medium surrounding the nanoparticle.

\section{Detailed balance energy emission to the SPP longitudinal near field}

We have seen that the semiclassical approach to the radiation absorption probability rate is correct within the approximations used, but the same is not verified for the case of the emission rate obtained with Eq. (17). A detailed balance approach will now be employed to calculate the radiation emission.

Let us consider the electronic state $|\lambda\rangle$ of higher energy that decays to the electronic state $|\mu\rangle$ of lower energy. For thermal equilibrium, the emission rate is $r_{\lambda \rightarrow \mu}=f_{\lambda}(1$ $\left.-f_{\mu}\right) w_{\lambda, k \tau_{i} \rightarrow \mu, k \tau_{i}}^{(e)}$, where $f_{\lambda}=\left(\exp \left[\left(\epsilon_{\lambda}-\epsilon_{F}\right) / k T\right]+1\right)^{-1}$ with $\epsilon_{\lambda}$ and $\epsilon_{F}$ the energy level of the state and the Fermi level, 
TABLE I. Near field parameters for several MNP shapes. The values of semiaxes $a, b$, and $c$ are normalized by the largest dimension of the ellipsoid.

\begin{tabular}{lccccccc}
\hline \hline \multicolumn{1}{c}{ Axes } & Along & Sphere & $H E$-dish & $k$-cigar & $E K$-dish & $H k$-dish & $E$-cigar \\
\hline$a$ & $\boldsymbol{H}$ & 1 & 1 & 0.1 & 0.1 & 1 & 0.1 \\
$b$ & $\boldsymbol{k}$ & 1 & 0.1 & 1 & 1 & 1 & 0.1 \\
$c$ & $\boldsymbol{E}$ & 1 & 1 & 0.1 & 1 & 0.1 & 1 \\
$L_{c}=\Psi_{c}(0)$ & & 0.333 & 0.070 & 0.490 & 0.070 & 0.861 & 0.020 \\
$\lambda_{\max }(\mathrm{nm})$ & & 772 & 2015 & 567 & 2015 & 267.7 & 4227.1 \\
$\left|\Lambda_{a \max }\right|$ & 30.02 & 42.67 & 34.87 & 42.67 & 107.15 & 77.15 \\
{$\left[\left|\Lambda_{a \max }\right| \Psi_{c}(0)\right]^{2}$} & & 100.14 & 8.82 & 291.84 & 8.82 & 8507.2 & 2.45 \\
\hline \hline
\end{tabular}

respectively; and similar formulas for the state $\mu$. The absorption rate can be written in the semiclassical frame as $r_{\mu \rightarrow \lambda}=f_{\mu}\left(1-f_{\lambda}\right) w_{\mu, k \tau_{i} \rightarrow \lambda, k \tau_{i}}^{(a)}$.

In thermal equilibrium the absorption rate equals that of the emissions, thus $f_{\lambda}\left(1-f_{\mu}\right) w_{\lambda, k \tau_{i} \rightarrow \mu, k \tau_{i}}^{(e)}=f_{\mu}\left(1-f_{\lambda}\right)$ $\times w_{\mu, k \tau_{i} \rightarrow \lambda, k \tau_{i}}^{(a)}$ Taking into account that $v_{k}=(\exp [(\hbar \omega) / k T]$ $-1)^{-1}$ and that $\epsilon_{\lambda}-\epsilon_{\mu}=\hbar \omega$, we obtain

$$
\frac{w_{\lambda, \boldsymbol{k} \tau_{i} \rightarrow \mu, \boldsymbol{k} \tau_{i}}^{(e)}}{w_{\mu, \boldsymbol{k} \tau_{i} \rightarrow \lambda, k \tau_{i}}^{(a)}}=\frac{f_{\mu}\left(1-f_{\lambda}\right)}{f_{\lambda}\left(1-f_{\mu}\right)}=\exp \left[\left(\mathfrak{E}_{\lambda}-\mathfrak{E}_{\mu}\right) / k T\right]=\frac{\nu_{\boldsymbol{k}}+1}{\nu_{\boldsymbol{k}}}
$$

so that

$$
\begin{aligned}
w_{\lambda, k \tau_{i} \rightarrow \mu, k \tau_{i}}^{(e)}= & \frac{\pi e^{2} \omega\left(\nu_{\boldsymbol{k}}+1\right)}{V n^{2} \varepsilon_{0}}\left|\left\langle\lambda\left|\boldsymbol{\tau}_{i} \sum_{j} \boldsymbol{r}_{j} \Lambda_{c} \Psi_{c}\left(\xi_{i}\right)\right| \mu\right\rangle\right|^{2} \\
& \times \delta\left(\mathfrak{E}_{\mu}-\mathfrak{E}_{\lambda}+\hbar \omega\right)
\end{aligned}
$$

which matches Eq. (11) for emission of radiation photons, apart from the scattering factor $\Lambda_{c} \Psi_{c}\left(\xi_{i}\right)$ in the integrand of the matrix element similar to the case of energy absorption from the MNP field.

\section{DISCUSSION}

Table I presents data for MNPs of several shapes. We consider ellipsoids whose orthogonal semiaxes $(a, b, c)$ are, respectively, in the direction of the magnetic field $(\boldsymbol{H})$, the propagation vector $(\boldsymbol{k})$, and the electric field $(\boldsymbol{E})$, as indicated in the second column. The quantities of interest shown are the geometrical depolarization factor $L_{c}=\Psi_{c}(0)$, which is dimensionless and depends on the shape of the MNP but not on its size, and the maximum absolute values of the polarizability $\Lambda_{c}$ and of the quantity $\left[\left|\lambda_{c}(\lambda)\right| \Psi_{c}(0)\right]^{2}$, that corresponds to the absorption enhancement factor caused by the MNP scattering effect close to its surface.

The polarizability $\Lambda_{c}$ determines the dipole formed in the MNP when it is illuminated by the radiation. In the metal, the MNP dielectric function has a frequency-dependent imaginary part. For our purpose it is well approximated by the Drude-Zener model (p. 638 of Ref. 38; nomenclature modified)

$$
\varepsilon_{\mathrm{MNP}}(\omega)=1+\frac{i \omega_{P}^{2} \tau}{\omega(1-i \omega \tau)},
$$

where $\omega_{P}=\sqrt{n_{e} e^{2} / m \varepsilon_{0}}$ is the plasma frequency $\left(n_{e}\right.$ density of electrons available for conduction) and $\tau$ is the scattering time of the conduction electrons. For silver $\omega_{P}=11.72$ $\times 10^{15} \mathrm{~s}^{-1}$ and $\tau=2.85 \times 10^{-15} \mathrm{~s}$ (adjusted from data on $\mathrm{p}$. 621 of Ref. 18). As for the medium dielectric constant, the value $\varepsilon=11$ (GaAs) is taken. The absolute value of $\Lambda_{c}$ is represented in Fig. 1, as a function of the wavelength in vacuum, for several values of $L_{c}$; as well as the maximum absorption enhancement factor $\left[\left|\lambda_{c}(\lambda)\right| \Psi_{c}(0)\right]^{2}$. The position

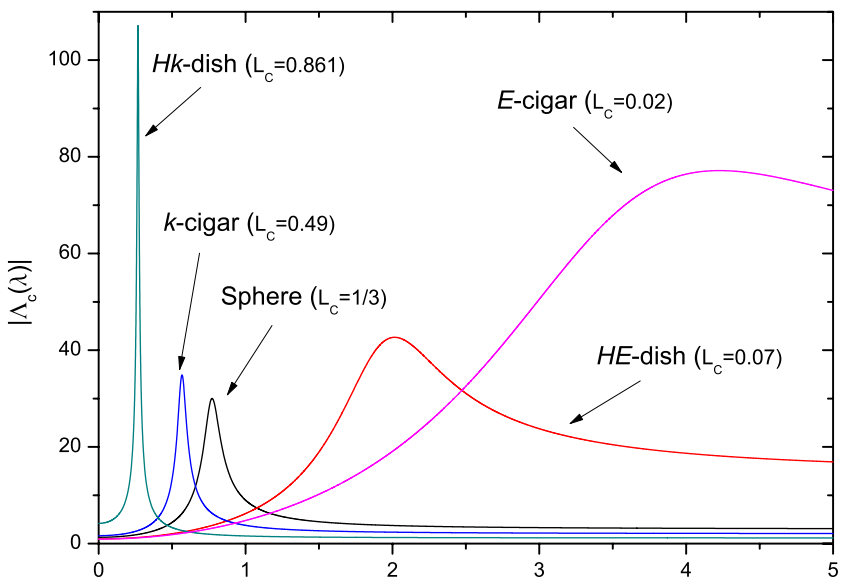

(a)

Wavelength $\lambda(\mu \mathrm{m})$

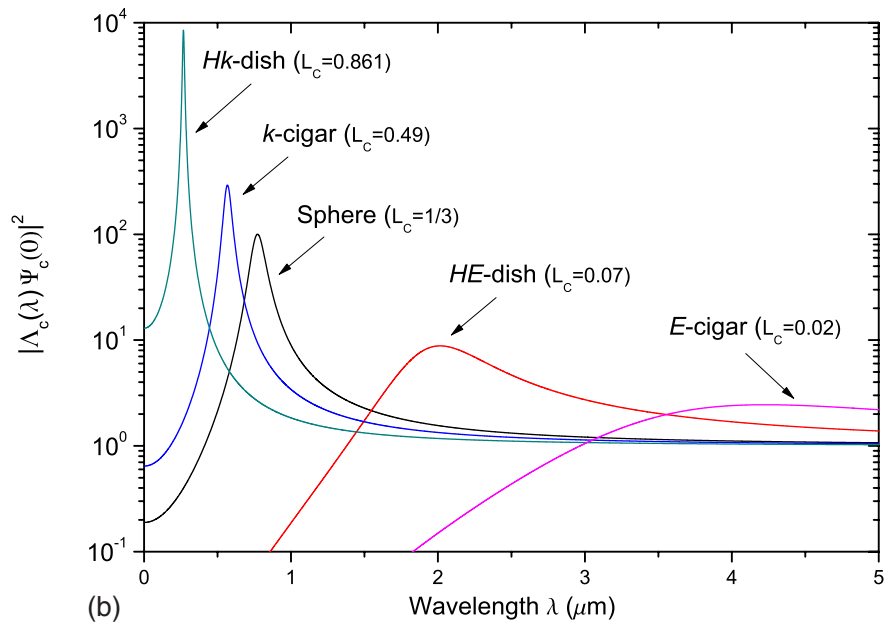

FIG. 1. (Color online) (a) Absolute value of the polarizability $\left|\Lambda_{c}(\lambda)\right|$ for different $L_{C}$ values as a function of the vacuum wavelength. (b) Plot of the absorption enhancement factor $\left[\left|\Lambda_{c}(\lambda)\right| \Psi_{c}(0)\right]^{2}$. 


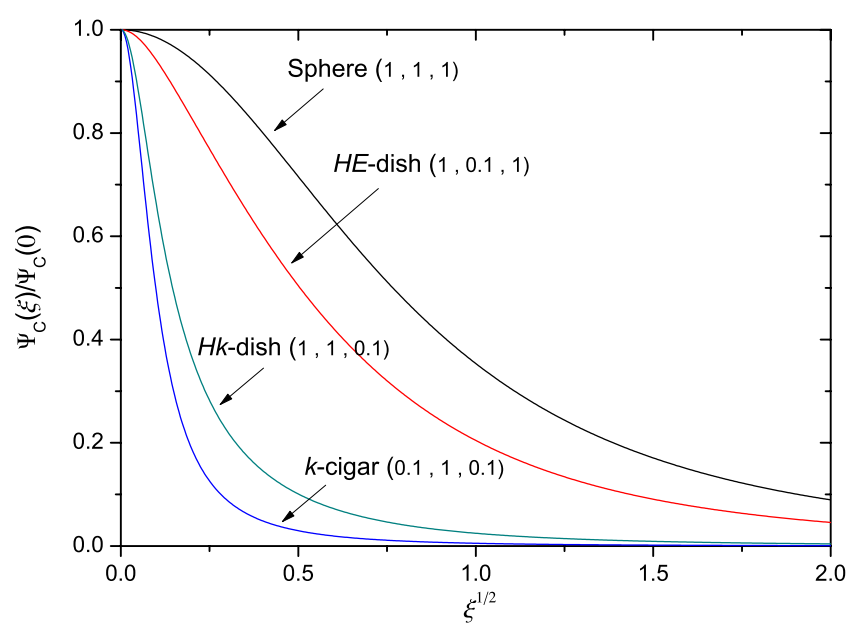

FIG. 2. (Color online) Plot of $\Psi_{c}$ as a function of $\xi^{1 / 2}$ for elliptical MNP of different semiaxes ratios $\left(a, b\right.$, and $c$ ) as indicated in Table I. The $\Psi_{c}$ curves are normalized to their corresponding maximum values $\left[\Psi_{c}(0)=L_{c}\right]$ at the ellipsoid surface. The horizontal axis $\left(\xi^{1 / 2}\right)$ is in units of the largest ellipsoid semiaxis.

and intensity of the maximum points are indicated in Table I.

The polarizability $\Lambda_{c}$ is independent of $\boldsymbol{r}$, therefore, this factor in the matrix element can go outside the integration in Eq. (26). The function $\Psi_{c}(\xi)$ depends on $\boldsymbol{r}$, therefore, in general, cannot be set as a factor. However, if the MNP size is taken to be much larger than the wavelength of the electron wavefunctions in the surrounding medium, then $\Psi_{c}(\xi)$ can be considered constant and also taken outside the matrix element integral. This is a reasonable assumption since the largest dimension of the MNP may be, in practice, some $100 \mathrm{~nm}$; whereas the electron wavefunctions in semiconductors are typically below $1 \mathrm{~nm}$. For electron wavefunctions associated to quantum dots, ${ }^{39}$ with dimensions of several tens of nm, it may not be accurate to take $\Psi_{c}(\xi)$ outside the matrix element.

The function $\Psi_{c}(\xi) / L_{C}$ is plotted in Fig. 2 versus $\xi^{1 / 2}$ to compare its actual extension [e.g., along the $y$ axis, $y=\left(b^{2}\right.$ $\left.+\xi)^{1 / 2}\right]$ for the selected MNP shapes. The horizontal axis is in units of the largest ellipsoid semiaxis. Figure 2 shows that the spherical MNP is the one with the largest absorption enhancement reach.

For a spherical MNP we can expect an absorption increase of 100 in the vicinity of the particle in the range of the near IR spectrum, and this can be useful for many applications. With elongated shapes in the direction of the incoming radiation ( $k$-cigar), with a bigger $\left|\Lambda_{c \text {,max }}\right|$, the gain is higher and the resonance is displaced to the visible but the distance at which the effects are noticeable is reduced. For a disk normal to the propagation vector ( $H E$-dish) the shape factor becomes smaller and the gain is also smaller; in this case the resonance is displaced further into the IR. All the three cases discussed are insensitive to the light polarization.

The highest gain in the table corresponds to a disk normal to the electric vector (Hk-dish). The gain can be very high with the resonance displaced to the UV. However, it is high only for the light properly polarized and it affects only those particles close to the disk.

\section{CONCLUSIONS}

Using a semiclassical approach we have deduced the expressions for transference of energy from a longitudinal field, such as the near field around a MNP sustaining a SPP, to a nondissipative (weakly absorbing) electronic system. This is compared to the absorption of ordinary transversal radiation.

The approach considers the near field as unaffected by the electronic system in the surroundings, although the perturbation of this near field is later deduced from detailed balance arguments. Our approach is verified by applying it to ordinary transversal radiation and comparing the results with the well-established photon absorption and emission rules.

It is found that in both expressions, the absorption of transversal radiation and of the longitudinal near field are closely related. For MNP that are much larger than the electronic systems the matrix element is just the same as for radiation multiplied by a factor that under favorable conditions, may be very large, almost $10^{4}$ with incoming polarized UV light. However, this enhancement value is highly dependent on the MNP shape and radiation wavelength. For practical cases, with a spherical MNP or one elongated in the direction of the propagation vector of the incoming radiation, the absorption increment can be well above 10 in the visible, and higher for the shorter wavelengths. For the absorption of longer wavelengths the SPP near field is still effective. Collective interaction with several MNP (Ref. 12) or maybe some other MNP shapes might be even more effective.

As mentioned in the introduction, to significantly improve the absorption of below-band-gap photons in quantum dot IBSCs a enhancement factor of at least one order of magnitude is necessary. Therefore, the near field amplification factors obtained here reveal that the described method is quite promissing to increase the photogenerated current in these cells.

\section{ACKNOWLEDGMENTS}

This work was supported by the European Commission within the project FULLSPECTRUM (SES6-CT-2003502620) and the projects NUMANCIA (S-0505/ENE/ 000310) funded by the Comunidad de Madrid, and GENESIS FV (CSD2006-0004) funded by the Spanish National Programme.

${ }^{1}$ E. Fermi, Rev. Mod. Phys. 4, 87 (1932).

${ }^{2}$ W. Heitler, The Quantum Theory of Radiation (Oxford University Press, Oxford, 1944).

${ }^{3}$ A. Messiah, Mécanique Quantique (Dunod, Paris, 1960).

${ }^{4}$ V. Berestetski, E. Lifchitz, and L. Pitayevski, Théorie Quantique Relativiste (Mir, Moscou, 1972).

${ }^{5}$ E. A. Power and S. Zienau, Philos. Trans. R. Soc. London, Ser. A 251, 427 (1959).

${ }^{6}$ J. J. Sanchezmondragon, N. B. Narozhny, and J. H. Eberly, Phys. Rev. Lett. 51, 550 (1983).

${ }^{7}$ A. Rahmani, P. C. Chaumet, and F. de Fornel, Phys. Rev. A 63, 023819 (2001).

${ }^{8}$ E. M. Purcell, Phys. Rev. 69, 681 (1946).

${ }^{9}$ E. Yablonovitch, Phys. Rev. Lett. 58, 2059 (1987).

${ }^{10}$ P. Lodahl, A. F. van Driel, I. S. Nikolaev, A. Irman, K. Overgaag, D. L. Vanmaekelbergh, and W. L. Vos, Nature (London) 430, 654 (2004).

${ }^{11}$ V. V. Klimov, M. Ducloy, and V. S. Letokhov, Eur. Phys. J. D 20, 133 (2002).

${ }^{12}$ L. A. Blanco and F. J. G. de Abajo, J. Quant. Spectrosc. Radiat. Transf. 89, 
37 (2004).

${ }^{13}$ G. Sun, J. B. Khurgin, and R. A. Soref, Appl. Phys. Lett. 90, 251105 (2007).

${ }^{14}$ D. V. Guzatov and V. V. Klimov, Phys. Rev. A 75, 052901 (2007).

${ }^{15}$ V. S. Zuev, Opt. Spectrosc. 102, 742 (2007).

${ }^{16}$ W. L. Barnes, A. Dereux, and T. W. Ebbesen, Nature (London) 424, 824 (2003).

${ }^{17}$ J. M. Pitarke, V. M. Silkin, E. V. Chulkov, and P. M. Echenique, Rep Prog. Phys. 70, 1 (2007).

${ }^{18}$ M. Born and E. Wolf, Principles of Optics (Pergamon, Oxford, 1975).

${ }^{19}$ C. F. Bohren and D. R. Huffman, Absorption and Scattering of Light by Small Particles (Wiley-VCH, Weinheim, 2004).

${ }^{20}$ Near-Field Optics and Surface Plasmon Polaritons, edited by S. Kawata (Springer-Verlag, Berlin, 2001).

${ }^{21}$ K. R. Catchpole and S. Pillai, J. Lumin. 121, 315 (2006).

${ }^{22}$ S. Pillai, K. R. Catchpole, T. Trupke, and M. A. Green, J. Appl. Phys. 101, 093105 (2007).

${ }^{23}$ Y. C. Chang, F. Y. Chou, P. H. Yeh, H. W. Chen, S. H. Chang, Y. C. Lan, T. F. Guo, T. C. Tsai, and C. T. Lee, J. Vac. Sci. Technol. B 25, 1899 (2007).

${ }^{24}$ C. Hagglund, M. Zach, G. Petersson, and B. Kasemo, Appl. Phys. Lett. 92, 053110 (2008)

${ }^{25}$ C. Hagglund, M. Zach, and B. Kasemo, Appl. Phys. Lett. 92, 013113 (2008).

${ }^{26}$ A. J. Morfa, K. L. Rowlen, T. H. Reilly, M. J. Romero, and J. van de
Lagemaat, Appl. Phys. Lett. 92, 013504 (2008).

${ }^{27}$ A. Luque and A. Marti, Phys. Rev. Lett. 78, 5014 (1997).

${ }^{28}$ A. Luque and A. Marti, Prog. Photovoltaics 9, 73 (2001).

${ }^{29}$ A. Martí, L. Cuadra, and A. Luque, Conference Record of the TwentyEighth IEEE Photovoltaic Specialists Conference, 2000, 940-943 (2000).

${ }^{30}$ A. Luque, A. Martí, C. Stanley, N. López, L. Cuadra, D. Zhou, and A. Mc-Kee, J. Appl. Phys. 96, 903 (2004).

${ }^{31}$ A. Luque, A. Marti, N. Lopez, E. Antolin, E. Canovas, C. Stanley, C. Farmer, L. J. Caballero, L. Cuadra, and J. L. Balenzategui, Appl. Phys. Lett. 87, 083505 (2005).

${ }^{32}$ A. Marti, E. Antolin, C. R. Stanley, C. D. Farmer, N. Lopez, P. Diaz, E. Canovas, P. G. Linares, and A. Luque, Phys. Rev. Lett. 97, 247701 (2006).

${ }^{33}$ A. Martí, E. Antolín, E. Cánovas, N. López, P. G. Linares, A. Luque, C. R. Stanley, and C. D. Farmer, Thin Solid Films 516 (2008).

${ }^{34}$ J. S. Biteen, L. A. Sweatlock, H. Mertens, N. S. Lewis, A. Polman, and H. A. Atwater, J. Phys. Chem. C 111, 13372 (2007).

${ }^{35}$ T. Härtling, P. Reichenbach, and L. M. Eng, Opt. Express 15, 12806 (2007).

${ }^{36}$ H. C. Casey and M. B. Panish, Heterostructure Lasers. Part A: Fundamental Principles (Academic, Orlando, FL, 1978).

${ }^{37}$ E. Kaxiras, Atomic and Electronic Structure of Solids (Cambridge University Press, New York, 2003).

${ }^{38}$ F. Seitz, Modern Theory of Solids (McGraw-Hill, New York, 1940).

${ }^{39}$ A. Marti, C. R. Stanley, and A. Luque, in Nanostructured Materials for Solar Energy Conversion, edited by T. Soga (Elsevier, Amsterdam, 2006). 